\title{
Analysis of Evolutionary Algorithms on Fitness Function with Time-linkage Property (Hot-off-the-Press Track at GECCO 2021)
}

\author{
Weijie Zheng \\ Guangdong Provincial Key \\ Laboratory of Brain-inspired \\ Intelligent Computation \\ Department of Computer Science and \\ Engineering \\ Southern University of Science and \\ Technology \\ Shenzhen, China
}

\author{
Huanhuan Chen \\ School of Computer Science and \\ Technology \\ University of Science and Technology \\ of China \\ Hefei, China
}

\author{
Xin Yao \\ Guangdong Provincial Key \\ Laboratory of Brain-inspired \\ Intelligent Computation \\ Department of Computer Science and \\ Engineering \\ Southern University of Science and \\ Technology \\ Shenzhen, China
}

\begin{abstract}
In real-world applications, many optimization problems have the time-linkage property, that is, the objective function value relies on the current solution as well as the historical solutions. Although the rigorous theoretical analysis on evolutionary algorithms has rapidly developed in the last two decades, it remains an open problem to theoretically understand the behaviors of evolutionary algorithms on time-linkage problems. This paper takes the first step towards the rigorous analyses of evolutionary algorithms for time-linkage functions. Based on the basic OneMax function, we propose a timelinkage function where the first bit value of the last time step is integrated but has a different preference from the current first bit. We prove that with probability $1-o(1)$, randomized local search and $(1+1)$ EA cannot find the optimum, and with probability $1-o(1)$, $(\mu+1)$ EA is able to reach the optimum.

This paper for the Hot-off-the-Press track at GECCO 2021 summarizes the work "Analysis of Evolutionary Algorithms on Fitness Function with Time-linkage Property" by W. Zheng, H. Chen, and $X$. Yao, which has been accepted for publication in the IEEE Transactions on Evolutionary Computation 2021 [19].
\end{abstract}

\section{CCS CONCEPTS}

- Theory of computation $\rightarrow$ Theory and algorithms for application domains; Theory of randomized search heuristics.

This work was supported by Guangdong Basic and Applied Basic Research Foun dation (Grant No. 2019A1515110177), Guangdong Provincial Key Laboratory (Grant No. 2020B121201001), the Program for Guangdong Introducing Innovative and Enterpreneurial Teams (Grant No. 2017ZT07X386), Shenzhen Science and Technology Program (Grant No. KQTD2016112514355531), the Program for University Key Laboratory of Guangdong Province (Grant No. 2017KSYS008), National Natural Science Foundation of China (Grant No. 61976111), and Science and Technology Innovation Committee Foundation of Shenzhen (Grant No. JCYJ20180504165652917). (Corresponding author: Xin Yao)

Weijie Zheng is also with School of Computer Science and Technology, University of Science and Technology of China, Hefei, China.

Xin Yao is also with CERCIA, School of Computer Science, University of Birmingham, Birmingham, United Kingdom.

Permission to make digital or hard copies of part or all of this work for personal or classroom use is granted without fee provided that copies are not made or distributed for profit or commercial advantage and that copies bear this notice and the full citation on the first page. Copyrights for third-party components of this work must be honored For all other uses, contact the owner/author(s).

GECCO '21 Companion, July 10-14, 2021, Lille, France

(c) 2021 Copyright held by the owner/author(s)

ACM ISBN 978-1-4503-8351-6/21/07.

https://doi.org/10.1145/3449726.3462725

\section{KEYWORDS}

Evolutionary algorithms, time-linkage, convergence, running time analysis.

\section{ACM Reference Format:}

Weijie Zheng, Huanhuan Chen, and Xin Yao. 2021. Analysis of Evolutionary Algorithms on Fitness Function with Time-linkage Property (Hot-off-thePress Track at GECCO 2021). In 2021 Genetic and Evolutionary Computation Conference Companion (GECCO '21 Companion), fuly 10-14, 2021, Lille, France. ACM, New York, NY, USA, 3 pages. https://doi.org/10.1145/3449726. 3462725

\section{SUMMARY OF OUR RESULTS}

Evolutionary Algorithms (EAs), one category of stochastic optimization algorithms that are inspired by the Darwinian principle and natural selection, have been widely utilized in real-world applications. However, the theoretical understandings are far behind the practical usage due to the difficulty of their mathematical analysis caused by their stochastic and iterative nature. Rigorous analyses can help to fundamentally understand EAs and ultimately design efficient algorithms in practice. Despite the increasing attention and insightful theoretical analyses in recent decades [1, 4, 7, 13, 21], there remain many important open areas that have not been considered in the evolutionary theory community.

One kind of important open issues is about the time-linkage problems. Time-linkage problems, firstly introduced by Bosman [2] into the evolutionary computation community, are the optimization problems where the objective function to be optimized relies not only on the solutions of the current time but also the historical ones. In other words, the current decisions also influence the future. There are plenty of applications with the time-linkage property, see more than 30 real-world (continuous and discrete) applications in the survey of [15].

The time-linkage optimization problems can be tackled offline or online according to different situations. If the problem pursues an overall solution with sufficient time budget and time-linkage dynamics can be integrated into a static objective function, then the problem can be solved offline. However, in the theoretical understanding on the static problem $[1,4,7,13,21]$, no static benchmark function in the evolutionary theory community is time-linkage.

Another situation that real-world applications often encounter is that the solution must be solved online as time goes by. This timelinkage online problem is a dynamic optimization problem [15]. As 
pointed out in [15], the whole evolutionary community, not only the evolutionary theory community, is lacking research on these real-world problems. The dynamic problems analyzed so far in the theory community majorly includes Dynamic OneMax [6], Magnitude and Balance [17], Maze [9], Bi-stable problem [8], dynamic linear functions [11], and the dynamic BinVal function [10] for dynamic pseudo-Boolean function, and dynamic combinatorial problems including the single-destination shortest path problem [12], makespan scheduling [14], the vertex cover problem [16], subset selection [18], graph coloring [3], etc. However, there is no theoretical analysis on dynamic time-linkage fitness functions, even no dynamic time-linkage pseudo-Boolean functions is proposed for the theoretical analysis.

In this work, we conduct the first step towards the understanding of EAs on the time-linkage function. When solving a time-linkage problem with EAs in an offline mode, the first thing faced by the practitioners utilizing EAs is how to encode the solution. There are obviously two straightforward encoding ways. Take the objective function relying on solutions of two time steps as an example. One way is to merely ignore the time-linkage dependency by solving a non-time-linkage function with double problem size. The other way is to consider the time-linkage dependency, encode the solution with the original problem size, but store the solutions generated in the previous time steps for the fitness evaluation. When solving the time-linkage problem in an online mode, engineers need to know before they conduct experiments whether the algorithm they use can solve the problem or not. Hence, in this paper, we design a time-linkage toy function based on OneMax to shed some light on these questions. This function, called $\operatorname{OneMax}_{\left(0,1^{n}\right)}$ where $n$ is the dimension size, is the sum of two components, one is the OneMax fitness of the current $n$-dimensional solution, the other one is the value of the first dimension in the previous solution but multiplying the opposite of the dimension size. The design of this function considers the situation when the current solution prefers a different value from the previous solution, which could better show the influence of different encodings. Also, it could be the core element of some dynamic time-linkage functions and used in the situation that each time step we only optimize the current state of the online problem in a limited time, so that the analysis of this function could also show some insights to the undiscovered theory for the dynamic time-linkage functions.

For our results, we analyze the theoretical behaviors of randomized local search (RLS) and two most common benchmark EAs, $(1+1) \mathrm{EA}$ and $(\mu+1) \mathrm{EA}$, on $\operatorname{OneMax}_{\left(0,1^{n}\right)}$. We show that with probability $1-o(1)$, RLS and $(1+1)$ EA cannot find the optimum of $\operatorname{OneMax}_{\left(0,1^{n}\right)}$ while the not small population size in $(\mu+1)$ EA can help it reach the optimum with probability $1-o(1)$. We also show that conditional on an event with probability $1-o(1)$, the expected runtime for $(\mu+1)$ EA is $O(n \mu)$.

Discussion: Here we discuss the reason for the searching difficulty of the $(1+1)$ EA in a more intuitive way. For the problems with no time-linkage property, most EAs use the global operators, which ensures the reachability of each search point in the search space, thus ensures the global convergence. One example for the not convergent EA could be the binary differential evolution analyzed in [5, Sec. 3.1] since the stochastic dependence results in its operators not global. For our case, it seems that the $(1+1)$ EA uses a global mutation operator. However, noting that the optimum is defined in an $(n+1)$-dimensional space while the search space is $n$-dimensional, the mutation operator in the $n$-dimensional space is not a global operator with respect to the $(n+1)$-dimensional space, thus could not ensure the global convergence. Besides, due to the selection operator, the $(1+1)$ EA will get stuck in some subspace and our results show that it happens with $1-o(1)$ probability. For the $(\mu+1)$ EA with the not small parent population size, our results show that with probability of $1-o(1)$, its maintained diversity will prevent the stagnation cases taking over the whole population before the optimum is reached.

Impact: This work makes the first attempt to the theoretical analysis of the EAs on time-linkage problem. It has brought some interesting results, like the theoretically positive support for the non-elitist evolutionary algorithms [20]. More theoretical discussions on more complicated and practical time-linkage problems will be addressed in the future.

\section{REFERENCES}

[1] Anne Auger and Benjamin Doerr. 2011. Theory of Randomized Search Heuristics: Foundations and Recent Developments. World Scientific.

[2] Peter A. N. Bosman. 2005. Learning, anticipation and time-deception in evolutionary online dynamic optimization. In GECCO 2005, Workshop Proceedings. ACM, 39-47.

[3] Jakob Bossek, Frank Neumann, Pan Peng, and Dirk Sudholt. 2019. Runtime analysis of randomized search heuristics for dynamic graph coloring. In GECCO 2019. ACM, 1443-1451.

[4] Benjamin Doerr and Frank Neumann. 2020. Theory of Evolutionary Computation: Recent Developments in Discrete Optimization. Springer Nature.

[5] Benjamin Doerr and Weijie Zheng. 2020. Working principles of binary differential evolution. Theoretical Computer Science 801 (2020), 110-142.

[6] Stefan Droste. 2002. Analysis of the (1+1) EA for a dynamically changing onemaxvariant. In CEC 2002, Vol. 1. IEEE, 55-60.

[7] Thomas Jansen. 2013. Analyzing Evolutionary Algorithms: The Computer Science Perspective. Springer Science \& Business Media.

[8] Thomas Jansen and Christine Zarges. 2015. Analysis of randomised search heuristics for dynamic optimisation. Evolutionary Computation 23, 4 (2015), 513-541.

[9] Timo Kötzing and Hendrik Molter. 2012. ACO beats EA on a dynamic pseudoboolean function. In PPSN 2012. Springer, 113-122.

[10] Johannes Lengler and Jonas Meier. 2020. Large population sizes and crossover help in dynamic environments. In PPSN 2020. Springer, 610-622.

[11] Johannes Lengler and Ulysse Schaller. 2018. The (1+1)-EA on noisy linear functions with random positive weights. In SSCI 2018. IEEE, 712-719.

[12] Andrei Lissovoi and Carsten Witt. 2015. Runtime analysis of ant colony optimization on dynamic shortest path problems. Theoretical Computer Science 561 (2015), 73-85.

[13] Frank Neumann and Carste Witt. 2010. Bioinspired Computation in Combinatorial Optimization - Algorithms and Their Computational Complexity. Springer, Berlin, Heidelberg.

[14] Frank Neumann and Carsten Witt. 2015. On the runtime of randomized local search and simple evolutionary algorithms for dynamic makespan scheduling. In IfCAI 2015. AAAI Press, 3742-3748.

[15] Trung Thanh Nguyen. 2011. Continuous dynamic optimisation using evolutionary algorithms. Ph.D. Dissertation. University of Birmingham.

[16] Mojgan Pourhassan, Wanru Gao, and Frank Neumann. 2015. Maintaining 2approximations for the dynamic vertex cover problem using evolutionary algorithms. In GECCO 2015. ACM, 903-910.

[17] Philipp Rohlfshagen, Per Kristian Lehre, and Xin Yao. 2009. Dynamic evolutionary optimisation: an analysis of frequency and magnitude of change. In GECCO 2009. ACM, 1713-1720.

[18] Vahid Roostapour, Aneta Neumann, Frank Neumann, and Tobias Friedrich. 2019. Pareto optimization for subset selection with dynamic cost constraints. In $A A A I$ 2019. 2354-2361.

[19] Weijie Zheng, Huanhuan Chen, and Xin Yao. 2021. Analysis of evolutionary algorithms on fitness function with time-linkage property. IEEE Transactions on Evolutionary Computation (2021). https://doi.org/10.1109/TEVC.2021.3061442

[20] Weijie Zheng, Qiaozhi Zhang, Huanhuan Chen, and Xin Yao. 2021. When nonelitism meets time-linkage problems. In GECCO 2021. ACM. Accepted.

[21] Zhi-Hua Zhou, Yang Yu, and Chao Qian. 2019. Evolutionary Learning: Advances in Theories and Algorithms. Springer. 